\title{
Passivity-based Control of Underactuated Biped Robots within Hybrid Zero Dynamics Approach
}

\author{
Hamid Sadeghian $^{1}$, Christian Ott ${ }^{2}$, Gianluca Garofalo ${ }^{2}$, and Gordon Cheng ${ }^{3}$
}

\begin{abstract}
The concept of hybrid zero dynamics is a promising approach for designing exponentially stabilizing controllers for dynamic walking with some degrees of underactuation. By this approach a feedback controller is designed such that a stable periodic orbit, within an invariant submanifold for the hybrid closed-loop system is created. This is usually achieved through an exponentially fast dynamics transverse to the zero dynamics manifold and the stability properties of such periodic orbit is then transferred to the full-order dynamic system. In this paper a passivity-based controller for a planar biped with one degree of underactuation is designed. By this approach we aim to preserve the natural dynamics of the system in the transverse dynamics (i.e. the dynamics transverse to the zero dynamics manifold) in contrast to the common inputoutput linearization method which cancels these dynamics. A Lyapunov stability analysis of the full-order system based on the conditional stability theorem is presented. By this analysis, the asymptotic stability of the periodic orbit in lower dimensional state space is extended to the full dimensional space. The results of the analysis are verified by simulation on a seven-link biped robot walking with zero ankle torque in sagittal plane.
\end{abstract}

\section{INTRODUCTION}

Biped robots with uncontrolled ankle joints or limited foot support let us to study dynamic locomotion with underactuated motion phases. Mainly heel strike, heel roll and toe roll phases need to be allowed as part of the gait design for robots. The degree of underactuation also increases when compliant elements are used in the structure of the biped robots to improve the energy efficiency. Beside the problems related to the underactuation, an additional challenge in biped robots arises from the impulsive events that happen when the swing leg impacts the ground. All of these issues make the underactuated biped robots a very challenging and rich problem because of its multi-phase, hybrid and periodic nature.

The Hybrid Zero Dynamics (HZD) is an extension of the zero dynamics concept to a hybrid system. In HZD the invariant manifold on which the zero dynamics is defined must be also invariant under the impact map of the system. It was introduced as a feedback controller for underactuated bipedal robots in [1]. In summary, a set of virtual constraints is first defined and a desired state-dependent trajectory that create stable periodic orbit in HZD manifold is then obtained.

This work was partially supported by the Helmholtz Association ( $\mathrm{VH}-$ NG-808).

${ }^{1}$ Engineering Department, University of Isfahan, Isfahan, Iran (Email: h.sadeghian@eng.ui.ac.ir)

${ }^{2}$ Institute of Robotics and Mechatronics, German Aerospace Center (DLR), Wessling, Germany

${ }^{3}$ Institute for Cognitive Systems, Faculty of Electrical Engineering and Information Technology, Technical University of Munich, Munich, Germany
The feedback design is finally completed by rendering the zero dynamics manifold finite time attractive. The method has had numerous successful implementations on bipeds with different degrees of underactuation [2]-[5]. Most of the works in the literature of HZD consider underactuated biped robots with point feet. However, the framework has been also extended to fully actuated flat-foot bipeds to exploit ankle torque [6] or to allow inclusion of phases with foot rotation and direct regulation of Zero Moment Point (ZMP) in walking gait design [7], [8]. For a comprehensive overview of the related approaches, [9] is referred.

In the earlier work of [1], finite-time convergence to the zero dynamics manifold was assumed. Later, this condition was relaxed to the exponential convergence with sufficient rate through an input-output linearization of the dynamics transverse to the zero dynamics manifold [10]. Most of the works that utilize HZD controller, realize this closed-loop structure on the output [3]-[5]. The motivation for assuring a sufficiently fast rate of exponential convergence is because of the expansive behavior of the impact maps which can lead to the divergence of the hybrid constrained dynamics. Hence, the convergence of the continuous dynamics to the manifold must be sufficiently attractive to overcome this behavior. Even though Control Lyapunov Function (CLF) approach is taken in [11] to expand the set of feedback controllers instead of simple PD controller, yet the core requirement is intact; a sufficiently fast-rate exponential convergence, with similar bounds to the linear case.

In this paper a passivity-based dynamics is devised as the transverse dynamics towards HZD submanifold. Unlike the common input-output linearization method, the passivitybased controller keeps the natural dynamics of the system which may enhance the performance of the system in terms of robustness and control effort [12], [13]. Similar objective is also followed in [14]. The stability analysis of the system is performed using theory of semi-definite Lyapunov functions. Sufficient conditions are established under which the stability of the periodic orbits in HZD submanifold is extended to the full order state space. The stability theory of semi-definite Lyapunov functions has been applied successfully to the analysis of control of redundant robots as well as orbital stabilization by the authors [13], [15]. Note that most of the existing locomotion researches are based on Poincaré map and sensitivity analysis [1], [3], [10], [16]. Due to the need to compute the whole solution during the analysis, only little analytical insight about the characteristics of the system (for instance, in terms of energy) is obtained. This reduces the flexibility for the development of new control algorithms. 


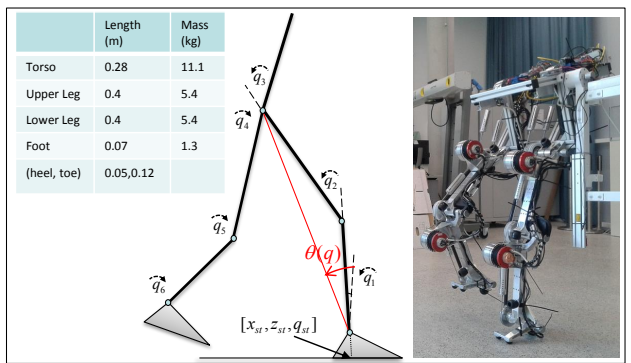

Fig. 1: Left: A seven-link planar biped robot with the choice of generalized coordinates designated based on stance foot. The system is underactuated when zero torque is given to the stance foot's ankle. Right; Compliant planar leg under development at DLR as our experimental testbed.

\section{TECHNICAL BACKGROUND}

\section{A. An Underactuated Planar Biped Robot}

An underactuated planar biped robot is naturally a hybrid system with impulse effects. It is comprising of a torso and two identical legs connected to the torso through the hip, as illustrated in Fig. 1. The following main assumptions are made through the paper;

- The gait is symmetric and constrained to sagittal plane;

- Walking consists of two alternative phases of motion; single support and instantaneous double support.

- The swing foot contacts the ground flat-footed;

- The impact is perfectly plastic (no rebound or slippage);

- The foot-ground friction is sufficient to prevent sliding;

- The ankle torque at the stance foot is set to zero and thus the model has one degree of underactuation.

The dynamics of an underactuated biped in single support phase is written by

$$
D(q) \ddot{q}+C(q, \dot{q}) \dot{q}+g(q)=B u, B=\left[\begin{array}{l}
0 \\
I_{m \times m}
\end{array}\right],
$$

where $q$ is $(n \times 1)$ set of angular coordinates of the system, $D(q)$ is the inertia matrix, $C(q, \dot{q})$ contains centrifugal/Coriolis terms and $g(q)$ is the vector of gravitational forces. $u$ is $(m \times 1)$ vector of input torques. The generalized coordinate $q$ can be partitioned to the un-actuated and actuated parts as $q=\left[\begin{array}{ll}q_{u}^{T} & q_{a}^{T}\end{array}\right]^{T}$. The configuration variable $q_{u}$ usually provides absolute orientation of the robot with respect to an inertial frame, and $q_{a}$ are measured with respect to the previous body frames. It is shown that the inertia matrix is independent of $q_{u}$ and thus, $D(q)=D\left(q_{a}\right)$ [17].

The above dynamics is followed until a rigid impact between the swing foot and the ground happens. At this time, while the biped configuration does not change (only a relabeling happens), the generalized velocities undergo a jump. The impact model $\Delta$ is obtained by relating the angular momentum before and after the impact as

$$
\left(q^{+}, \dot{q}^{+}\right)=\Delta_{q, \dot{q}}\left(q^{-}, \dot{q}^{-}\right),
$$

where superscripts $(.)^{-}$and $(.)^{+}$refer to the quantities right before and after the impact, respectively.

\section{B. System with Impulse Effects}

A nonlinear autonomous system with impact map can be written as

$$
\Sigma: \begin{cases}\dot{x}=f(x), & x^{-} \notin \mathcal{S}, \\ x^{+}=\Delta\left(x^{-}\right), & x^{-} \in \mathcal{S},\end{cases}
$$

where the state manifold $\chi$ is a subset of $\mathbb{R}^{n}$, and $f$ is a vector field on $\chi$. The impact switching surface $\mathcal{S}$ is a one-dimensional surface $\mathcal{S}$ which is defined as $\mathcal{S}=$ $\{x \in \chi \mid H(x)=0\}$, where $H: \chi \rightarrow \mathbb{R}$ is $C^{1}$, and $\forall x \in \mathcal{S}, \partial H / \partial x(x) \neq 0 . \Delta$ is the impact model and can be considered as an instantaneous re-initialization of the state of the system when the system trajectory intersects $\mathcal{S}$.

The following definition, taken from [10], will help us to constitute the problem in the following sections based on a well-defined mathematics framework.

Definition 1: For autonomous system $\Sigma$, if a $C^{1}$ embedded submanifold $\mathcal{Z} \subset \chi$ is hybrid invariant (invariant under both continuous and impact dynamics [10]) and $\mathcal{S} \cap \mathcal{Z}$ is $C^{1}$ with one dimension less than that of $\mathcal{Z}$, then

$$
\Sigma_{\mathcal{Z}}: \begin{cases}\dot{z}=f_{\mathcal{Z}}(z), & z^{-} \notin \mathcal{S} \cap \mathcal{Z}, \\ z^{+}=\Delta_{\mathcal{S} \cap Z}\left(z^{-}\right), & z^{-} \in \mathcal{S} \cap \mathcal{Z},\end{cases}
$$

is called a hybrid restriction dynamics of the autonomous system $\Sigma$. $f_{\mathcal{Z}}$ and $\Delta_{\mathcal{S} \cap \mathcal{Z}}$ are the restrictions of $f$ and $\Delta$ to $\mathcal{Z}$ and $\mathcal{S} \cap \mathcal{Z}$, respectively.

\section{HZD OF UNDERACTUATED BIPED: A PASSIVITY-BASED APPROACH}

In this section a passivity-based controller is designed as the transverse dynamics for a planar underactuated biped robot. By this controller we aim to preserve the natural inertia of the system in the closed-loop dynamics which increases the robustness of the system and decreases the control effort [12], [13].

In the context of HZD, as a common approach, a set of virtual constraints $y=h(q) \in \mathbb{R}^{m}$ in the form of output is first defined. The error of these outputs are then zeroed by an input-output linearization controller. This controller inverts the dynamics associated with the virtual constraints and thus cancels the natural dynamics of the system which is practically not recommended (for normal HZD development please see [17]). It is argued that the natural dynamics of the system is applied by cost function minimization in the virtual constraint for low control effort [9]. Nevertheless, the possible application of passivity-based controllers that are avoiding a full feedback inversion are much appealing. In the following a passivity-based HZD controller is developed. For the sake of comparison, at first the HZD controller with an exponential rate of convergence is developed.

The dynamics (1) can be separated as,

$$
\left\{\begin{array}{l}
D_{11} \ddot{q}_{u}+D_{12} \ddot{q}_{a}+\underbrace{C_{11} \dot{q}_{u}+C_{12} \dot{q}_{a}+g_{1}}_{\Omega_{1}}=0, \\
D_{21} \ddot{q}_{u}+D_{22} \ddot{q}_{a}+\underbrace{C_{21} \dot{q}_{u}+C_{22} \dot{q}_{a}+g_{2}}_{\Omega_{2}}=u .
\end{array}\right.
$$


This equations can be equivalently re-written in the following form

$$
\left\{\begin{array}{l}
\ddot{q}_{u}=-D_{11}^{-1}\left(D_{12} \ddot{q}_{a}+\Omega_{1}\right), \\
\underbrace{\left(D_{22}-D_{21} D_{11}^{-1} D_{12}\right)}_{\bar{D}\left(q_{a}\right)} \ddot{q}_{a} \underbrace{-D_{21} D_{11}^{-1} \Omega_{1}+\Omega_{2}}_{\bar{\Omega}(q, \dot{q})}=u .
\end{array}\right.
$$

\section{A. Partial Feedback Linearization Approach}

A partial feedback controller

$$
u=\bar{D} \nu+\bar{\Omega}
$$

is applied to convert the system (6) to simple form

$$
\left\{\begin{array}{l}
\ddot{q}_{u}=-D_{11}^{-1}\left(D_{12} \nu+\Omega_{1}\right) \\
\ddot{q}_{a}=\nu
\end{array}\right.
$$

Starting from this system, one can design $\nu$ such that the actuated joints follow a desired state-dependent trajectory. Lets define parametric polynomials $h_{d}^{\alpha}(\theta)$ which its parameters $\alpha=\alpha^{*}$ are obtained based on boundary conditions on the configuration and velocity at the beginning and at the end of a step to impose a periodic motion on hybrid submanifold. $\theta=\theta(q)$ is a monotonically increasing quantity which is used to substitute time in parametrization of the periodic motion. The angle of the line that connects the stance foot to the hip is a good measure for $\theta$ (Fig. 1). Defining the error $y=q_{a}-h_{d}^{\alpha^{*}}(\theta)$, the control command

$$
\nu=\ddot{h}_{d}^{\alpha^{*}}(\theta, \dot{\theta}, \ddot{\theta})+\frac{k_{d}}{\epsilon} \dot{y}+\frac{k_{p}}{\epsilon^{2}} y
$$

results in

$$
\ddot{y}+\frac{k_{d}}{\epsilon} \dot{y}+\frac{k_{p}}{\epsilon^{2}} y=0,
$$

which exponentially regulates the error $y$ (transverse variables vector) to zero with sufficient rate of convergence adjusted by tuning parameter $\epsilon>0$. The rest of the dynamics, given by the first equation, is actually the internal dynamics of the system. Without loss of generality, consider a planar biped with one degree of underactuation. In this case the coordinate $q_{u}$ can be chosen as $q_{u}=\theta$, and the swing phase zero dynamics is given by the first equation in (8), upon substitution of (9), considering $y$ and $\dot{y}$ equal to zero, i.e.,

$$
\left[D_{11}+D_{12}\left(\frac{\partial h_{d}^{\alpha^{*}}}{\partial \theta}\right)\right] \ddot{\theta}+D_{12} \frac{\partial^{2} h_{d}^{\alpha^{*}}}{\partial \theta^{2}} \dot{\theta}^{2}+\Omega_{1}=0
$$

The set $\mathcal{Z}:=\{x=(q, \dot{q}) \mid y=0, \dot{y}=0\}$, is then the zero dynamics submanifold of the full dimensional state space. Considering perfect tracking of the error $y$, the parameters in $h_{d}^{\alpha}$ are obtained such that a stable periodic orbit is realized by the zero dynamics (11) as well as by discrete impact dynamics (2) to satisfy invariance condition $\Delta(\mathcal{S} \cap \mathcal{Z}) \subset \mathcal{Z}$. Such set of parameters $\alpha^{*}$, corresponding to a stable periodic motion with acceptable domain of attraction, are obtained through optimization (see Section V).

\section{B. Passivity-based Approach}

Starting from (6), the following passivity-based command is proposed

$$
u=\bar{D} \ddot{h}_{d}^{\alpha^{*}}-\left(\left(\bar{C}+k_{d}\right) \dot{y}+k_{p} y\right)+\bar{\Omega},
$$

where $\bar{C}$ is obtained such that $\dot{\bar{D}}=\bar{C}+\bar{C}^{T}$. To obtain such matrix, first notice that, $\bar{D}=D_{22}-D_{21} D_{11}^{-1} D_{12}$ is symmetric. Assume that the Coriolis/centrifugal matrix $C$ is chosen such that $\dot{D}=C+C^{T}$. The derivative of $\bar{D}$ is obtained as

$\dot{\bar{D}}=\dot{D}_{22}-\dot{D}_{21} D_{11}^{-1} D_{12}-D_{21}\left(\frac{d}{d t}\left(D_{11}^{-1}\right) D_{12}-D_{11}^{-1} \dot{D}_{12}\right)$.

Note that, $\frac{d}{d t}\left(D_{11}^{-1}\right)=-D_{11}^{-1} \dot{D}_{11} D_{11}^{-1}$, and thus,

$$
\begin{aligned}
\dot{\bar{D}} & =\dot{D}_{22}-\dot{D}_{21} D_{11}^{-1} D_{12}+D_{21} D_{11}^{-1} \dot{D}_{11} D_{11}^{-1} D_{12} \\
& -D_{21} D_{11}^{-1} \dot{D}_{12} \\
& =C_{22}+C_{22}^{T}-\left(C_{21}+C_{12}^{T}\right) D_{11}^{-1} D_{12} \\
& +D_{21} D_{11}^{-1}\left(C_{11}+C_{11}^{T}\right) D_{11}^{-1} D_{12}-D_{21} D_{11}^{-1}\left(C_{12}+C_{21}^{T}\right) \\
& =\underbrace{C_{22}-C_{21} D_{11}^{-1} D_{12}}_{A_{1}}+C_{22}^{T}-D_{21} D_{11}^{-1} C_{12} \\
& \underbrace{-C_{12}^{T} D_{11}^{-1} D_{12}}_{A_{2}}-D_{21} D_{11}^{-1} C_{21}^{T}+\underbrace{D_{21} D_{11}^{-1} C_{11} D_{11}^{-1} D_{12}}_{A_{3}} \\
& +D_{21} D_{11}^{-1} C_{11}^{T} D_{11}^{-1} D_{12} \\
& =A_{1}+A_{1}^{T}+A_{2}+A_{2}^{T}+A_{3}+A_{3}^{T} .
\end{aligned}
$$

Finally, $\bar{C}=A_{1}+A_{2}+A_{3}$.

Applying (12), the closed-loop of the system for actuated variables is

$$
\bar{D} \ddot{y}+\left(\bar{C}+k_{d}\right) \dot{y}+k_{p} y=0 .
$$

In comparison with (10), the above closed-loop dynamics of transverse variables keep specially the inertia matrix of the system in the closed-loop error dynamics. Clearly, the system is nonlinear due to the use of inertia matrix. By the above dynamics, the error $y$ is expected to go to zero asymptotically. The crucial point is the structure of such dynamics which is not as in (10). The behavior of the whole system also depends on the internal dynamics which is obtained from first equation in (6) substituting $\ddot{q}_{a}$ from (15). In summary, the closed-loop of the system are given by the following equations

$$
\left\{\begin{array}{l}
{\left[D_{11}+D_{12}\left(\frac{\partial h_{d}^{\alpha^{*}}}{\partial \theta}\right)\right] \ddot{\theta}+D_{12} \frac{\partial^{2} h_{d}^{\alpha^{*}}}{\partial \theta^{2}} \dot{\theta}^{2}} \\
\left.-D_{12} \bar{D}^{-1}\left(\left(\bar{C}+k_{d}\right) \dot{y}+k_{p} y\right)\right)+\Omega_{1}=0,(\theta, y)^{-} \notin \mathcal{S} \\
\bar{D} \ddot{y}+\left(\bar{C}+k_{d}\right) \dot{y}+k_{p} y=0, \\
(\theta, \dot{\theta}, y, \dot{y})^{+}=\Delta\left((\theta, \dot{\theta}, y, \dot{y})^{-}\right), \quad(\theta, y)^{-} \in \mathcal{S} .
\end{array}\right.
$$

Note that the hybrid zero dynamics of this system is the same as the case where linear exponential regulator was used (Equation (11)). This can be verified by setting $y$ and $\dot{y}$ to zero in (16). 


\section{LyAPUNOV-BASED STABILITY ANALYSIS}

\section{A. General Discussion}

The exponential convergence of the transverse dynamics, is just a sufficient condition to keep the eigenvalues sufficiently small (c.f. Corollary 4 in [10]). The aim of this section is to provide sufficient conditions for the structure of the closed-loop dynamics to make the periodic orbit $\mathcal{O}$, stable and attractive for full-order system.

The stability analysis is mainly based on the conditional stability theorem proposed in [18] for analysis of the equilibrium points. Recently, it has been extended to the analysis of invariant sets, including limit cycles [15]. We also use some useful concepts related to the stability of the switched systems, which relies on multiple Lyapunov functions [19]. Both of the theorems are reviewed briefly in the following.

Theorem 2: (Conditional Stability Theorem for Invariant Set) [15] let $\Omega$ be an invariant set for the system $\dot{x}=$ $f(x)$, and let $V(x)$ be a continuous function defined in $B_{v}(\Omega) \subset \chi$ such that $V(x) \geq 0, \forall x \in B_{v}(\Omega), V(\Omega)=0$ and $\dot{V}(x) \leq 0, \forall x \in B_{v}(\Omega)$. If $\Omega$ is asymptotically stable conditionally to the largest positively invariant set $M^{*}$ within $M=\left\{x \in B_{v}(\Omega) \mid \dot{V}(x)=0\right\}$, then $\Omega$ is asymptotically stable.

Theorem 3: (Multiple Lyapunov Functions Theorem) [20] Consider a switched system $\dot{x}=f_{i}(x(t)), i=1, \ldots, N$, with each $f_{i}$ globally Lipschitz continuous and $f_{i}(0)=0$. Suppose that we have candidate Lyapunov functions $V_{i}(x(t))$. let $\mathcal{S}$ be the set of all switching sequences associated with the system. The system is stable if,

- $\dot{V}_{i}(x) \leq 0$, when the $\mathrm{i}$-th subsystem is active.

- $V_{i}$ is decreasing on the "switched on" time sequence of the i-th subsystem.

Consider the system $\Sigma$ given by (3). Suppose that the following hypotheses are met;

1) There exist global coordinates $x=(z, \eta)$ for $x \in \mathbb{R}^{n}$, such that $z \in \mathbb{R}^{k}$ and $\eta \in \mathbb{R}^{n-k}$ and $f(x)$ has the form

$$
f(x)=\left[\begin{array}{l}
f_{1}(z, \eta) \\
f_{2}(\eta)
\end{array}\right] .
$$

2) For $\mathcal{Z}:=\{(z, \eta) \in \chi \mid \eta=0\}, \mathcal{S} \cap \mathcal{Z}$ is a $(k-1)$ dimensional submanifold of $\mathcal{Z}$, and it is hybrid invariant, i.e. $\Delta(\mathcal{S} \cap \mathcal{Z}) \subset \mathcal{Z}$.

3) System $\Sigma$ has an asymptotically stable periodic orbit $\mathcal{O}$ contained in $\mathcal{Z}$ and transverse to $\mathcal{S}$.

4) The function $V(\eta)>0$, positive definite in $\eta$, locally around $\Omega=\mathcal{O}$ exists that satisfies the following conditions;

- It is decreasing along the continuous part of the dynamics, $\dot{V}(\eta) \leq 0$, and $V(\Omega)=0$.

- The sequence of values of $V\left(\eta_{i}\right)$, where $\eta_{i}$ are measured right after the impact, i.e. on the surface $\Delta\left(S_{i}\right)$, is a decreasing sequence. In other words, on switch to the continuous part of the dynamics, $V(\eta)$ has less energy than the last switch on.
The above Hypotheses are similar to the ones that are considered in Theorem 4.6 in [17] for time scale separation of the transverse dynamics and the HZD. The main difference is that instead of Lyapunov conditions in Hypothesis 4, $f_{2}(\eta)$ is given as a linear dynamics depending on parameter $\epsilon>0$ such that, $f_{2}(\eta)=A(\epsilon) \eta$, and $\lim _{\epsilon \rightarrow 0} e^{A(\epsilon)}=0$.

The following proposition is given.

Proposition 4: Under the Hypothesis 1-4, the asymptotic stability of a periodic orbit of the hybrid restricted dynamics is transferred to the full-order system.

Sketch of Proof: $\mathcal{Z}$ is hybrid invariant and it means that starting on $\mathcal{Z}$, the states stay on $\mathcal{Z}$ even after the impact. Thus, $\Omega=\mathcal{O}$ is an invariant set for the full-order system $\Sigma$. By Hypothesis 4, there exists $V(\eta)>0$, such that $\dot{V}(\eta) \leq 0$ along the trajectory of the system. Note that, the function vanishes on the invariant set $\Omega$ since on the orbit, $\eta=0$. The situation provided by the Hypothesis 4 resembles the conditions in Theorem 3, where a discrete Lyapunov like function exists for the system. Note that this candidate may not include all the variables of the system and thus it is positive semi-definite. Following the conditional stability theorem, $\Omega=\mathcal{O}$ is asymptotically stable conditionally to the largest positively invariant set $M^{*}=\mathcal{Z}$ within $M=\left\{x \in B_{v}(\Omega) \mid \dot{V}(\eta)=0\right\}$ (by Hypothesis 3) and thus by conditional stability theorem, $\Omega=\mathcal{O}$ is asymptotically stable.

Remark 6: It can be shown that the function $f_{2}(\eta)$ in Hypothesis 1 can be replaced by $f_{2}(z, \eta)$ provided that $f_{2}(z, 0)=0$. In this case the previous results are still hold if one can find $V(z, \eta) \geq 0$, (positive definite in $\eta$ ) that satisfies the conditions in Hypothesis 4.

Note that using above proposition, it is still necessary to check the asymptotic stability of the orbit in lower dimensional space. This can be performed by a Poincaré map analysis of the system in the zero dynamics manifold.

\section{B. Stability Analysis of Passivity-based Controller}

Lets go back to the closed-loop structure (16). Based on the notation of the previous section, $z=(\theta, \dot{\theta})$ and $\eta=(y, \dot{y})$ are the states of zero dynamics and transverse dynamics, respectively. For this system the Hypothesis 13 in Proposition 4 is already satisfied regarding Remark 6. Consider semi-positive definite function

$$
V(y, \dot{y}, \theta)=\frac{1}{2} \dot{y}^{T} \bar{D}(y, \theta) \dot{y}+\frac{1}{2} y^{T} k_{p} y .
$$

The time derivative of (17) is given by

$$
\begin{aligned}
\dot{V} & =\dot{y}^{T}\left(-\left(\bar{C}+k_{d}\right) \dot{y}-k_{p} y\right)+\frac{1}{2} \dot{y}^{T} \dot{\bar{D}} \dot{y}+\dot{y}^{T} k_{p} y, \\
& =-\dot{y}^{T} k_{d} \dot{y} \leq 0,
\end{aligned}
$$

which is negative semi-definite along the continuous part of the system trajectory. The closed-loop structure of transverse variables reminds the closed-loop of PD+ controller. In [21] a strict Lyapunov function has been introduced. As it can be seen in the next section from some values of $k_{p} \geq \bar{k}_{p}$ and $k_{d} \geq \bar{k}_{d}$, the second condition of the Hypothesis 4 can 
also be satisfied. Thus by Proposition 4, an asymptotically stable periodic orbit of the hybrid zero dynamics is also asymptotically stable for the full-order system.

\section{Simulation Results}

For the purpose of this study a seven-link planar biped robot is chosen. The parameters of the robot are based on a compliant planar robot currently under development at DLR (Fig. 1). Each degree of freedom is independently driven by a DC motor through a mechanism of cable, pulley and spring [22]. A set of generalized coordinates $q=$ $\left[x_{s t}, z_{s t}, q_{s t}, q_{1}, \ldots, q_{6}\right]^{T}$ is assigned to the system starting from the stance foot as depicted in Fig. 1.

Bezier polynomials of order 3 are chosen to define the virtual constraints. They are expressed as functions of the variable $\theta=-q_{1}-q_{2} / 2$. This variable is chosen as the angle of the hip with respect to the vertical reference.

The ankle torque at stance leg is set to zero to obtain a periodic orbit of the system. The ankle of the swing leg is controlled similar to the other actuated joints, following a Bezier polynomial with proper boundary conditions. The evolution of the underactuated variable is obtained from the zero dynamics. The search for a periodic motion is cast as a constrained nonlinear optimization problem. The final configuration and velocity $\left(q_{f}^{*}, \dot{q}_{f}^{*}\right)$ is found such that, the solution is periodic and the integral-squared torque per step length, $J=1 / L \int_{0}^{T}\left\|u^{*}\right\|^{2} d t$, is minimized. $u^{*}$ is the torque required to keep the system on the orbit and can be calculated from (12) considering $y$ and $\dot{y}$ equal to zero. This minimization is performed subject to appropriate constraints and is not mentioned here for the sake of brevity [17]. After this constrained, offline optimization, a fixed-point solution $x^{*}=\left(q_{f}^{*}, \dot{q}_{f}^{*}\right) \in \mathcal{S} \cap \mathcal{Z}$ corresponding to a periodic motion is obtained. Note that the coefficients of Bezier polynomials are uniquely related to this fixed-point [3].

In order to verify the proposed approach, the controller (12) is used, starting from an initial condition off the periodic orbit. The phase plane related to zero dynamics states as well as the Lyapunov function (17) accompany with the position and velocity errors are depicted in Fig. 2 for a proper set of gains. The discontinuity at the impact is manifested as a straight line. The decreasing behavior of this function during the continuous phase of the dynamics as well as the discontinuous phase (in consecutive impacts) are clearly seen. The evolution of $q_{i}$ in the phase plane which are convergent to periodic orbits are not shown for brevity. The values related to the step length, step period and average velocity of the biped are reported in Fig. 3. As it was discussed in the previous section, the behavior of the transverse dynamics is crucial to the overall stability of the orbit. Fig. 4 illustrates the same plot as in Fig. 2 with less gains. $V$ increases after each impact and thus does not satisfy the conditions of the Lyapunov function in the Hypothesis 4 of the proposed Proposition 4. As a consequence the system trajectory diverges.

Finally a comparison is made between the controller (7) and (9) and the passivity-based controller (12). Because
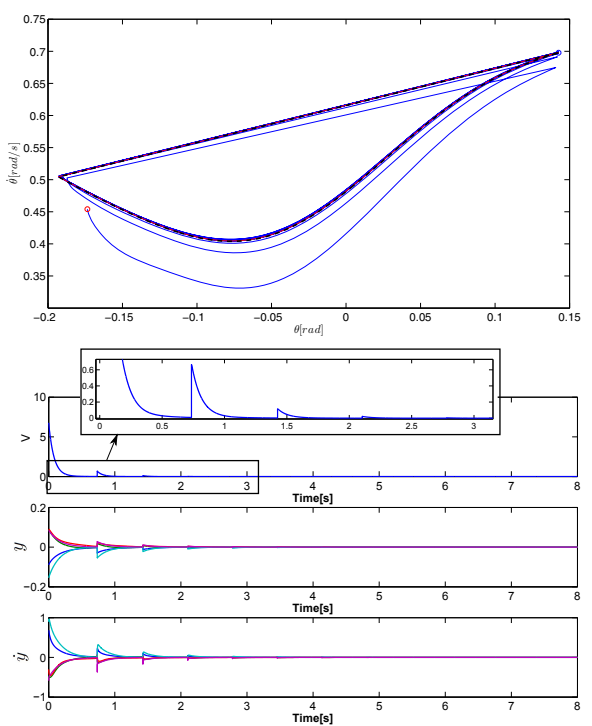

Fig. 2: (Top:) Phase plane $\theta-\dot{\theta}$, for the passivity-based controller (12) with the parameters $\overline{k_{p}}=120 I, \overline{k_{d}}=20 I$. It finally converges (red line) to the orbit $\mathcal{O}$ (black dash). (Down:) Lyapunov function (17), and error $y$ and $\dot{y}$.

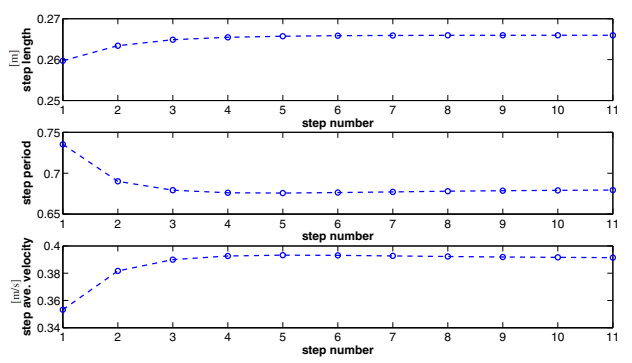

Fig. 3: step length, step period and average velocity corresponding to the parameters in Fig. 4.
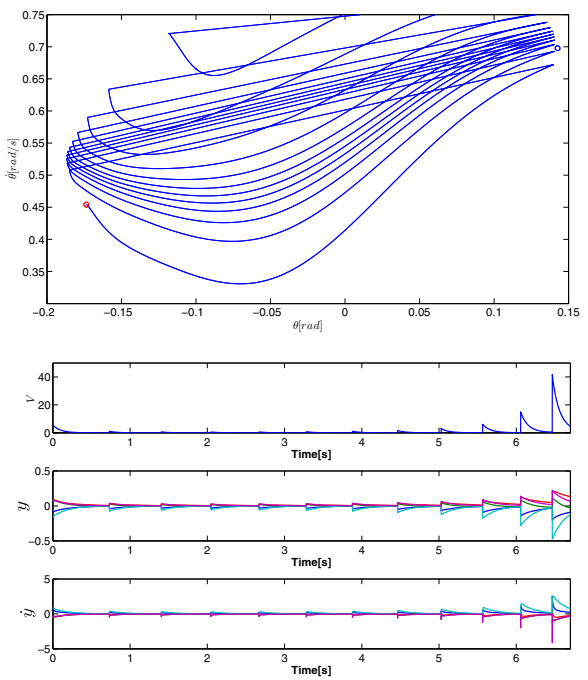

Fig. 4: (Top:) Phase plane for $\theta-\dot{\theta}$, for the passivitybased controller associated with the parameters $\overline{k_{p}}=100 I$, $\overline{k_{d}}=20 I$. The system states diverges gradually in this case. (Down:) Lyapunov function (17), and error $y$ and $\dot{y}$. 

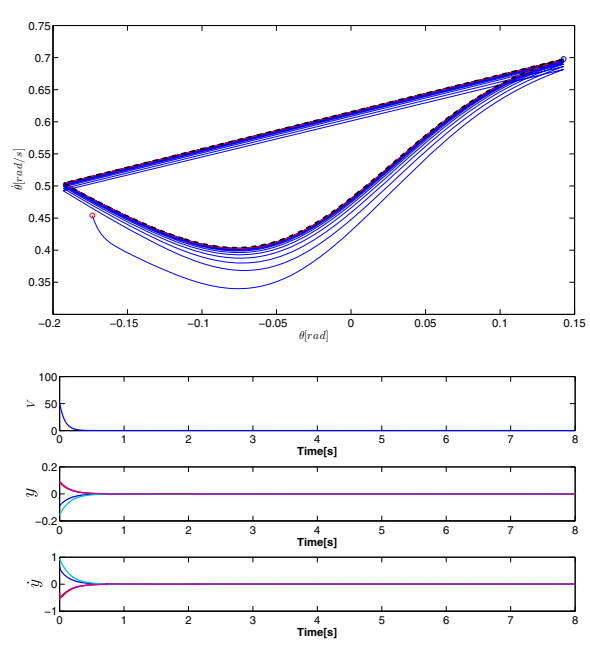

Fig. 5: (Top:) Phase plane for $\theta-\dot{\theta}$, for the linearization controller given by (7) and (9) with the parameters $k_{p} / \epsilon^{2}=\operatorname{diag}\{600,600,600,1200,900\}, k_{d} / \epsilon=$ $\operatorname{diag}\{100,100,100,200,150\}$. It finally converges to the orbit $\mathcal{O}$ (depicted by black dash). The red line shows the convergent orbit of the system. (Down:) Lyapunov function $V(\eta)=\frac{1}{2} \dot{y}^{T} \dot{y}+\frac{1}{2} y^{T} k_{p} y$, and error $y$ and $\dot{y}$.

the nature of two controllers are different with different closed-loop structures, the gains in (9) is selected as follows. The numerical value of $\bar{D}^{-1}$ in a generic configuration is calculated and its diagonal elements are gathered in a diagonal matrix. The gains $k_{p}$ and $k_{d}$ are then obtained by multiplication of this matrix with $\overline{k_{p}}, \overline{k_{d}}$. Based on this selection, the simulation is performed and the results are illustrated in Fig. 5. While the system rapidly converges to the zero dynamics manifold, the convergence to the orbit $\mathcal{O}$ takes longer than the passivity-based controller in Fig. 2.

\section{CONCLUSION}

The stabilization of periodic orbits of the hybrid zero dynamics relies mainly on the input-output linearization of the transverse variables with exponential and sufficient rate of convergence. The main proposition of this paper aims to alter this condition on the transverse dynamics in terms of Lyapunov function. Having a stable periodic orbit in an invariant zero dynamics manifold, sufficient conditions for the structure of the closed-loop dynamics is proposed to make the periodic orbit, stable and attractive for the fullorder system. This enables us to use a nonlinear passivitybased controller and as a consequence a natural transverse dynamics toward the hybrid zero dynamics manifold. The proposed approach was effectively verified by simulation on a seven-link planar biped robot.

\section{ACKNOWLEDGMENT}

The first author would like to thank Dr. Marion Leibold from LSR lab at TUM, for general discussion on the stability of the hybrid systems and hybrid zero dynamics.

\section{REFERENCES}

[1] E. R. Westervelt, J. W. Grizzle, and D. E. Koditschek, "Hybrid zero dynamics of planar biped walkers," Automatic Control, IEEE Transactions on, vol. 48, no. 1, pp. 42-56, 2003.

[2] E. R. Westervelt, G. Buche, and J. W. Grizzle, "Experimental validation of a framework for the design of controllers that induce stable walking in planar bipeds," The International Journal of Robotics Research, vol. 23, no. 6, pp. 559-582, 2004.

[3] C. Chevallereau, J. W. Grizzle, and C.-L. Shih, "Asymptotically stable walking of a five-link underactuated 3-d bipedal robot," Robotics, IEEE Transactions on, vol. 25, no. 1, pp. 37-50, 2009.

[4] A. Ramezani, J. W. Hurst, K. A. Hamed, and J. Grizzle, "Performance analysis and feedback control of atrias, a three-dimensional bipedal robot," Journal of Dynamic Systems, Measurement, and Control, vol. 136, no. 2, p. 021012, 2014.

[5] S. Veer, M. S. Motahar, and I. Poulakakis, "On the adaptation of dynamic walking to persistent external forcing using hybrid zero dynamics control," in Intelligent Robots and Systems (IROS), 2015 IEEE/RSJ International Conference on. IEEE, 2015, pp. 997-1003.

[6] H. Sadeghian, C. Ott, and G. Cheng, "Exploiting ankle torque for orbital stabilization in biped robots; a hybrid zero dynamics approach," in Humanoid Robots (Humanoids), 2016 IEEE-RAS 16th International Conference on. IEEE, 2016, pp. 870-875.

[7] J. H. Choi and J. Grizzle, "Planar bipedal walking with foot rotation," in Proceedings of the 2005, American Control Conference, 2005. IEEE, 2005, pp. 4909-4916.

[8] C. Chevallereau, D. Djoudi, and J. W. Grizzle, "Stable bipedal walking with foot rotation through direct regulation of the zero moment point," IEEE Transactions on Robotics, vol. 24, no. 2, pp. 390-401, 2008.

[9] J. W. Grizzle, C. Chevallereau, R. W. Sinnet, and A. D. Ames, "Models, feedback control, and open problems of $3 \mathrm{~d}$ bipedal robotic walking," Automatica, vol. 50, no. 8, pp. 1955-1988, 2014.

[10] B. Morris and J. W. Grizzle, "Hybrid invariant manifolds in systems with impulse effects with application to periodic locomotion in bipedal robots," Automatic Control, IEEE Transactions on, vol. 54, no. 8, pp. 1751-1764, 2009.

[11] A. D. Ames, K. Galloway, K. Sreenath, and J. W. Grizzle, "Rapidly exponentially stabilizing control lyapunov functions and hybrid zero dynamics," Automatic Control, IEEE Transactions on, vol. 59, no. 4, pp. 876-891, 2014.

[12] C. Ott, Cartesian impedance control of redundant and flexible-joint robots. Springer, 2008

[13] H. Sadeghian, L. Villani, M. Keshmiri, and B. Siciliano, "Task-space control of robot manipulators with null-space compliance," Robotics, IEEE Transactions on, vol. 30, no. 2, pp. 493-506, 2014.

[14] O. Montano, Y. Orlov, Y. Aoustin, and C. Chevallereau, "Orbital stabilization of an underactuated bipedal gait via nonlinear $h \infty$-control using measurement feedback," Autonomous Robots, pp. 1-19, 2016.

[15] G. Garofalo, C. Ott, and A. Albu-Schaffer, "Orbital stabilization of mechanical systems through semidefinite lyapunov functions," in American Control Conference (ACC), 2013. IEEE, 2013, pp. 57155721.

[16] K. A. Hamed and J. W. Grizzle, "Event-based stabilization of periodic orbits for underactuated 3-d bipedal robots with left-right symmetry," Robotics, IEEE Transactions on, vol. 30, no. 2, pp. 365-381, 2014.

[17] E. R. Westervelt, J. W. Grizzle, C. Chevallereau, J. H. Choi, and B. Morris, Feedback control of dynamic bipedal robot locomotion. CRC press, 2007, vol. 28.

[18] A. Iggidr, B. Kalitine, and R. Outbib, "Semidefinite lyapunov functions stability and stabilization," Mathematics of Control, Signals and Systems, vol. 9, no. 2, pp. 95-106, 1996.

[19] D. Liberzon, Switching in systems and control. Springer Science \& Business Media, 2012.

[20] M. S. Branicky, "Multiple lyapunov functions and other analysis tools for switched and hybrid systems," Automatic Control, IEEE Transactions on, vol. 43, no. 4, pp. 475-482, 1998.

[21] V. Santibáñez and R. Kelly, "Strict lyapunov functions for control of robot manipulators," Automatica, vol. 33, no. 4, pp. 675-682, 1997.

[22] F. Loeffl, A. Werner, D. Lakatos, J. Reinecke, S. Wolf, R. Burger, T. Gumpert, F. Schmidt, C. Ott, M. Grebenstein et al., "The dlr crunner: Concept, design and experiments," in Humanoid Robots (Humanoids), 2016 IEEE-RAS 16th International Conference on. IEEE, 2016, pp. 758-765. 